\title{
FINAL PROGRESS REPORT - GRANT \#DE-FG02-98ER62558
}

\section{P.I.: Temple F. Smith, Boston University}

It was proposed in the original aims of the proposal that an integrated set of gene sequence/function/structure analysis tools be assembled to support maximal extraction functional information from among the available complete genome Open Reading Frames (ORFs) and their probable regulatory regions. Following is the progress that was made on the three major components of the proposal.

\section{The construction of diagnostic functional patterns/profiles for all probable protein functional domains.}

1.a. The incorporation of a new prior-based profile procedure into the multidomain dissection procedures (Adams et al. Protein Science 5, 1996).

The lab developed a database of sequence domain profiles is composed of two divisions, one in conjunction with our analysis of mitochondrial imported proteins, and one based on the selection of taxonomically distant apparent homologs from among the current set of completed microbial genomes. In addition, we used the identified functional domains associated with the Pfam and PROSITE databases. All such profiles were used to search for matches among the more than 120,000 predicted proteins from the current completely sequenced genomes. One significant result was that $60 \%$ of our profiles match proteins containing at least one additional domain. In order to facilitate the use of this database both internally and externally, we have developed a Web graphic interface. In addition, a full relational database was installed to support $\mathrm{SQL}$, queries of the profiles, and associated data.

In addition to using the work of Adams and Das as originally proposed, we extended it to using local domain-matching Hidden Markov Models. The development of HMMs for multidomain protein analysis has continued. This work became the major component of a graduate student's doctoral thesis. One recent publication (Bienkowska et al. 2001) was in part the result of this continuing effort.

1.b. The development of simple cross-database annotation comparison procedures to allow for the development of a controlled profile annotation vocabulary with maximal synonym linkages.

\section{1.b.1. Development of graphic display tools for profile-induced multidomain, multisequence alignments and their annotation}

In conjunction with the analysis of mitochondrial proteins, we developed a web-based interface that summarizes the set of proteins matching a profile. We incorporated a graphics display of the annotation associated with each profile's microbe matched sequences. 'This

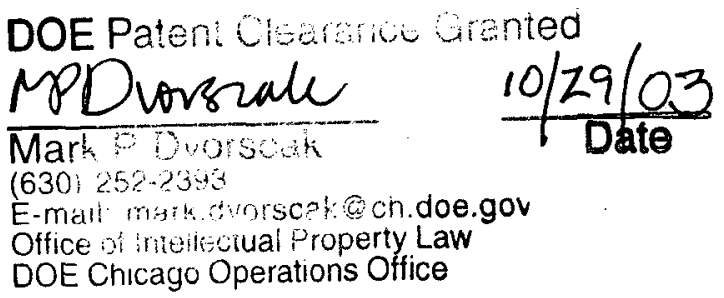




\section{DISCLAIMER}

This report was prepared as an account of work sponsored by an agency of the United States Government. Neither the United States Government nor any agency thereof, nor any of their employees, makes any warranty, express or implied, or assumes any legal liability or responsibility for the accuracy, completeness, or usefulness of any information, apparatus, product, or process disclosed, or represents that its use would not infringe privately owned rights. Reference herein to any specific commercial product, process, or service by trade name, trademark, manufacturer, or otherwise does not necessarily constitute or imply its endorsement, recommendation, or favoring by the United States Government or any agency thereof. The views and opinions of authors expressed herein do not necessarily state or reflect those of the United States Government or any agency thereof. 


\section{DISCLAIMER}

Portions of this document may be illegible in electronic image products. Images are produced from the best available original document. 
consists of a set of word nodes connected with a weighted edge. The weight is proportional to the number of times the two words appeared adjacent in the annotation set.

\section{1.b.2.. This grant contained a successful consortium with CIFN-UNAM in Mexico. The following was reported by Julio Collado-Vides:}

Research Project: "Microbial protein and regulatory analysis and database program"

Literature search:

We conducted a continuous search in original literature publications and added the information to the RegulonDB database. At the end of the proposal period this database contained on the order of 500 promoters, 500 regulatory interactions (with information on their binding sites and effect in transcription), and 400 operons. We made major progress on this goal, although it is an on-going goal that cannot be considered finished.

Genome Predictions:

A. Regulatory sites in $E$. coli and $H$. influenzae.

Based on information of known sites and the operons they regulate, we initiated a multigenome strategy to generate stronger predictions on several bacterial genomes. The computational recognition of regulatory sites was limited by their considerable variability in sequence. Another source of information used was orthology and orthology of operons.

Based on predictions of operons in other bacteria, we analyzed the CRP and FNR regulons in $E$. coli and $H$. influenzae. In this way, we generated a new way of evaluating predictions, where the strongest ones are those that show conservation of the DNA-binding motifs for the regulatory proteins upstream from the genes, and also show a conservation of operons in two bacteria. This work resulted in two publications.

\section{B. Prediction of operons}

By the use of RegulonDB, the database on transcription regulation in $E$. coli, we developed and published a method to predict transcription units in this organism (Salgado et al., PNAS 97, 2000). In this way all genes in the $E$. coli genome are clustered either into known operons or predicted operons. We estimate on the order of 800 total operons in $E$. coli.

\section{Other Microbial Genomes: Prediction of operons}

We have also demonstrated that the method used in the $E$. coli genome is also applicable to other prokaryotic genomes. Another part of our studies about transcription units made use of data in RegulonDB to show that genes within operons have a tendency towards conservation among genomes in the three domains of life. We found that such conservation occurs in the form of gene fusions in Eukarya, and that genes organized in operons in E. coli, when found together in other genomes, might indeed be in a single transcription unit there. This is on-going research. 
2. The development of methodologies needed to construct full hash tables and associated ORF distributions of all possible regulatory words. The development of sequence comparative tools to search for and identify regulatory circuits among sets of coordinately regulated genes.

Rather than attempting to use all possible regulatory words, we applied our method to a unique set of mRNA 3'-processing regulatory words. We developed a tool for the prediction of mRNA $3^{\prime}$-processing (cleavage and polyadenylation) sites in the yeast Saccharomyces cerevisiae, based on a discrete state-space model or hidden Markov model. Comparison of predicted sites with experimentally verified 3 '-processing sites indicates good agreement. All predicted or known yeast genes were analyzed to find probable 3 '-processing sites. Known alternative 3 '-processing sites, both within the $3^{\prime}$-untranslated region and within the protein coding sequence were successfully identified, leading to the possibility of prediction of previously unknown alternative sites. The lack of an apparent $3{ }^{\prime}$-processing site calls into question the validity of some predicted genes. This was specifically investigated for predicted genes with overlapping coding sequences.

3. The construction of prototype data structures, assembled (and updated) from existing databases and other data resources, to support the above developments and their validation.

3.a. The major goal to develop a prototype database of profiles for the functional/structural protein domains definable among the completely sequenced microbial genomes has been completed. We have generated over 2400 profiles from a defining set ranging from two to seven sequences involving 575 Gram-positive bacteria genes, 826 Gramnegative bacteria genes, 463 Archaea genes and 1480 Eukaryotic (mainly Yeast and $C$. elegans) genes. This set of profiles was searched against all completed genomes and the PDB database of determined protein structures. They matched functional domains with very high statistical significance, of 8,879 proteins among the 70,000 -plus protein sequences in the completed genome database. The search against the protein structure database resulted in over 300 matches with unquestionable statistical significance ( $Z$ scores greater than 40 !). These matches allowed us to associate directly the secondary structure to positions in the profiles.

We developed new full-length domain profiles as the list of completely sequenced genomes expanded. We also developed better priors for our Bayesian mixture approach to generate profiles from small sequence-defining sets. We also put major effort into comparing our profile match results with those of other approaches. For example, we generated a large number of profiles from the pfam-defining (HMM training) set of proteins. We found that a rather large fraction of these defining sets shared domains. Thus not all of the pfam patterns represented independent functional or evolutionary domains. This forced us to examine all of our own domain profiles for "overlap" in the common sequence regions represented. It helped in identifying profiles that were initially of sufficient length to span more than one domain. 
3.b. A second goal we completed is our world wide web site designed to make available the above information. A fully functional web site (bmerc-www.bu.edu) is currently running with a number of features. These include:

- A profile search engine that allows the external user either to search a sequence against all of our profiles, or search the profile library for functional keyword matches.

- For every sequence in our library of microbe proteins we have identified their probable homologs. This is continuously updated using both our own profiles and BLAST. Our measure of similarity is the percentage of effective identities, which for example had the following interpretation: $34 \%$ effective identities would mean that either there is $34 \%$ amino acid identities over the total length of the shorter of the two sequences, $100 \%$ over $34 \%$ of that length, or anything in between these two.

- These features are linked directly to the primary data sources when they are available on the world wide web. In addition we designed our web interface such that there is a machine-readable, down-loadable and table-formatted output option for nearly all search or analysis results.

Since a long-term goal of this supported research was to provide the functional genomic community with useful tools, we used our profiles in combination with various forms of protein structural information for very detailed analyses of a couple of important protein families.

In addition, this has been applied to the evolution of Mitochondrial functional protein domains. We were able to exploit our profile library and profile-matched domain dissection implication to explore the probable origins of the more than 420 proteins that go to make up the yeast mitochondria. Insofar as possible, all profiles were split on the basis of PDB structure information and/or apparent large profile alignment gaps. This allowed independent phylogenies to be constructed for each domain. The functionally related sets of genes were expected to display nearly identical phylogenetic relationships within each "functional" set, but potentially quite different relationships between sets. This is what is most often seen, and the analyses reveal a complex and fascinating history for the mitochondrial genes. This is a history not only in which horizontal transfer proceeded in both directions, but in which no simple history appears constructible for some well-studied proteins (Mohr et al. 2001). 


\section{Publications:}

Salgado, Heladia, Moreno-Hagelsieb, Gabriel, Smith, Temple F. and Collado-Vides, Julio. (2000). Operons in Escherichia coli: Genomic analyses and predictions. Proceedings of the National Academy of Sciences 97, 6652-6657.

Bienkowska, Jadwiga, Hongxian He and Temple F. Smith. Automatic pattern embedding in protein structure models. IEEE, Intell. Syst. 16: 21-25, 2001.

Moreno-Hagelsieb, Gabriel, Treviño, Victor, Pérez-Rueda, Ernesto, Smith, Temple F. and Collado-Vides, Julio. (2001). Transcription unit conservation in the three domains of life: a perspective from Escherichia coli. Trends in Genetics 17 (4), 175-177.

Plasterer, Thomas N., Smith, Temple F. and Mohr, Scott C. (2001). Survey of human mitochondrial diseases using new genomic/proteomic tools. Genome Biology 2(6).

Graber, Joel, McAllister, Gregory D. and Smith, Temple F. (2002). Probabilistic prediction of Saccharomyces cerevisiae mRNA 3'-processing sites. Nucleic Acids Research 30(8), 1851 1858.

He, Hongxian, McAllister, Gregory and Smith, Temple F. (2002). Triage protein fold prediction. PROTEINS 48, 654-663. 